Evolution, University of Arizona,

Tucson, Arizona 85721, USA

1. Jorde, L. B., Bamshad, M. \& Rogers, A. R. BioEssays 20, 126-136 (1998).

2. Majumder, P. P. Evol. Anthropol. 6, 100-110 (1998).

3. Bamshad et al. Hum. Biol. 68, 1-33 (1996).

4. Tambia, S. J. in The Character of Kinship (ed. Goody, J.) 191-229 (Cambridge Univ. Press, 1973)

5. Mantel, N. Cancer Res. 27, 209-220 (1967).

6. Heyer, E. et al. Hum. Mol. Genet. 6, 799-803 (1997).

7. Saitou, N. \& Nei, M. Mol. Biol. Evol. 4, 406-425 (1988).

8. Anderson et al. Nature 290, 457-465 (1981)

9. Wakeley, J. J. Mol. Evol. 37, 613-623 (1993).

10. Hammer, M. F. et al. Mol. Biol. Evol. 15, 427-441 (1998).

\section{Effects of progesterone or neuroactive steroid?}

Smith et al. ${ }^{1}$ reported some interesting results about the effects of progesterone treatment and withdrawal on the gene encoding the $\mathrm{GABA}_{\mathrm{A}}$ receptor $\alpha 4$ subunit, a constituent of receptors for the neurotransmitter GABA ( $\gamma$-aminobutyric acid). However, we disagree with their conclusion that the effects of progesterone withdrawal are mediated by reduced levels in the brain of the $\mathrm{GABA}_{\mathrm{A}}$ receptor active neurosteroid $3 \alpha$-OH- $5 \alpha$-pregnan-20-one $(3 \alpha, 5 \alpha$-THP, or allopregnanalone). The ability of indomethacin to reverse the effects of progesterone was interpreted as evidence that the effects of progesterone were mediated by $3 \alpha, 5 \alpha$-THP. Smith et al. ${ }^{1}$ did not provide direct evidence that levels of $3 \alpha, 5 \alpha$-THP were reduced by indomethacin (which reversed the effects of progesterone), or that progesterone levels were not altered by indomethacin. In some cases, indomethacin can increase progesterone levels directly ${ }^{2}$, which may explain why indomethacin can reverse the effects of progesterone withdrawal.

The effect of indomethacin blockade of $3 \alpha$-hydroxysteroid oxidoreductase activity is dependent on the relative levels of $3 \alpha, 5 \alpha-$ THP and its immediate precursor $5 \alpha$ dihydroprogesterone. When $3 \alpha, 5 \alpha$-THP concentrations are raised after progesterone infusion or stress, indomethacin would block the oxidation of $3 \alpha, 5 \alpha$-THP, increasing its concentration. Using the same dose regimen as ref. 1 we find that stress-induced elevation of $3 \alpha, 5 \alpha$-THP levels in the brain are increased by pretreatment with indomethacin (Fig. 1). Blockade of this enzyme with fluoxetine has also been shown to raise $3 \alpha, 5 \alpha$-THP levels in the rat brain $^{3}$. The omission of $3 \alpha, 5 \alpha-$ THP and progesterone levels in the report by Smith et $a l^{1}{ }^{1}$ therefore raises concerns about the conclusion that $3 \alpha, 5 \alpha$-THP mediates the effects of progesterone withdrawal.

Manipulation of progesterone levels in female rats is likely to influence the expression of many genes as well as the levels of

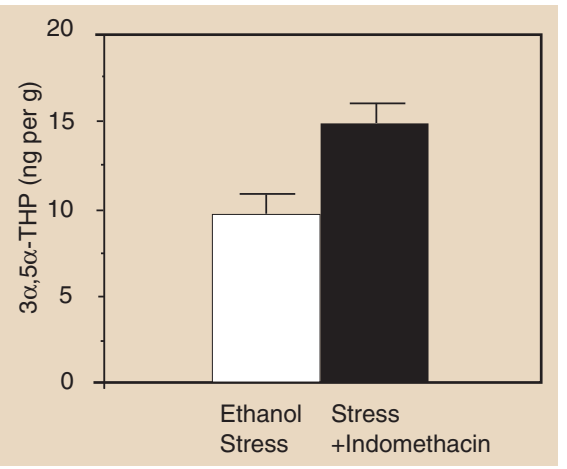

Figure 1 Indomethacin increases stress-induced $3 \alpha, 5 \alpha-$ THP levels in rat cerebral cortex. Male Sprague-Dawley rats were habituated to handling and injection. Indomethacin (0.1 mg per kg, intraperitoneal) was administered 20 minutes before ethanol injection stress ( $2 \mathrm{~g}$ per $\mathrm{kg}$, intraperitoneal) and $3 \alpha, 5 \alpha-$ THP levels were measured as described ${ }^{4}$. Indomethacin increased stress-induced $3 \alpha, 5 \alpha-7 H P$ levels by $58.5 \pm 62.0 \%(P<0.01$, Student's $t$-test $)$.

numerous steroids other than $3 \alpha, 5 \alpha$-THP. Therefore, even if $3 \alpha, 5 \alpha$-THP levels are reduced by indomethacin, this does not demonstrate that the effects of progesterone withdrawal on $\mathrm{GABA}_{\mathrm{A}}$ receptors are mediated by $3 \alpha, 5 \alpha$-THP. Correlational evidence, although suggestive, does not establish a causal relationship.

The effects of progesterone and ethanol withdrawal on $\mathrm{GABA}_{\mathrm{A}}$ receptor $\alpha 4$ subunit gene expression are similar. However, cross-tolerance between ethanol and $3 \alpha, 5 \alpha-$ THP does not occur in vivo. Indeed, chronic ethanol administration produces cross-tolerance to benzodiazepines, but also results in sensitization to both behavioural and neurochemical responses to $3 \alpha, 5 \alpha-\mathrm{THP}^{4}$. Moreover, this effect is greater in female than male rats, suggesting that the higher progesterone levels in female rats are associated with greater sensitization to $3 \alpha, 5 \alpha-$ $\mathrm{THP}^{5}$. This sensitization is probably due to intrinsic changes in $\mathrm{GABA}_{\mathrm{A}}$ receptors, as concentrations of $3 \alpha, 5 \alpha$-THP are not altered after chronic ethanol administration $^{4}$. Although the effects of ethanol ${ }^{4,6}$ and progesterone on $\mathrm{GABA}_{\mathrm{A}}$ receptor $\alpha 4$ gene expression are known, there is no direct evidence that $3 \alpha, 5 \alpha$-THP alters the expression of this gene.

\section{A. Leslie Morrow ${ }^{\star}$, Margaret J. VanDoren,} Leslie L. Devaud

Departments of Psychiatry and ${ }^{\star}$ Pharmacology, and Center for Alcohol Studies,

University of North Carolina School of Medicine, Chapel Hill, North Carolina 27599-7178, USA e-mail:morrow@med.unc.edu

Smith et al. reply - Morrow et al. are incorrect to state that we did not provide direct evidence that $3 \alpha-\mathrm{OH}-5 \alpha$-pregnan20 -one $(3 \alpha, 5 \alpha$-THP, or allopregnanolone) levels were reduced by indomethacin. We pointed out ${ }^{1}$ that our previous studies ${ }^{7}$ showed that indomethacin administration concomitant with progesterone reduced the level of $3 \alpha, 5 \alpha$-THP in the central nervous system from $8.2 \pm 1.3 \mathrm{ng}$ per $\mathrm{g}$ to $2.3 \pm$ $0.35 \mathrm{ng}$ per $\mathrm{g}$ without altering levels of $5 \alpha$ DHP or progesterone. This treatment, which prevented the withdrawal of $3 \alpha, 5 \alpha$ THP that normally accompanies progesterone withdrawal, completely prevented the withdrawal effects of progesterone we observed $^{1}$, including acceleration in the decay of GABA-gated current and upregulation of the $\mathrm{GABA}_{\mathrm{A}}$-receptor $\alpha 4$ subunit.

We have shown ${ }^{8}$ that direct withdrawal of $3 \alpha, 5 \alpha$-THP, in the absence of progesterone withdrawal, also results in faster decay of GABA-gated current in association with upregulation of the $\alpha 4$ subunit. In this case, withdrawal from the GABA-modulatory metabolite $3 \alpha, 5 \alpha$-THP was accomplished with the use of a $5 \alpha$-reductase inhibitor to block $3 \alpha, 5 \alpha$-THP formation without altering progesterone levels. Taken together, these results establish $3 \alpha, 5 \alpha$-THP as the active agent that exhibits withdrawal properties. In contrast, Morrow et al. administer indomethacin 20 minutes before ethanol stress, which would prevent direct conversion of $3 \alpha$-DHP to $3 \alpha, 5 \alpha$-THP. The indomethacin-induced increases in $3 \alpha, 5 \alpha$ THP levels they report after ethanol stress can be explained only by stress-induced effects on the degradation of $3 \alpha, 5 \alpha$-THP.

We further disagree with the suggestion by Morrow et al. that our findings are purely correlational. Indomethacin is a highly selective blocker of $3 \alpha$-hydroxysteroid oxidoreductase activity ${ }^{9}$, and so reduces $3 \alpha, 5 \alpha$ THP levels specifically, without altering levels of other hormones such as $5 \alpha$-DHP or progesterone. This is therefore the most definitive procedure to determine the role of the $3 \alpha, 5 \alpha$-THP metabolite in the upregulation of the $\alpha 4$ subunit we observe after progesterone withdrawal. Direct administration of $3 \alpha, 5 \alpha$-THP would be a less convincing model because back-conversion to $5 \alpha$-DHP can occur ${ }^{9}$. Furthermore, systemic administration of progesterone best mimics physiological conditions in our animal model of premenstrual syndrome, in which circulating progesterone is converted in the CNS to $3 \alpha, 5 \alpha-\mathrm{THP}^{10}$.

Earlier results from Morrow et al. indicate that sensitivity to $3 \alpha, 5 \alpha-\mathrm{THP}$ is increased after ethanol withdrawal ${ }^{4}$. We are intrigued by this finding because recent data from our laboratory indicate that withdrawal of $3 \alpha, 5 \alpha$-THP also increases ethanol potentiation of GABA-gated current in CA1 hippocampus by $71.2 \pm 5.4 \%$ over control levels. This result is consistent with reports of increased alcohol consumption in women during the premenstrual period. Both of these findings clearly distinguish the effects of ethanol from those of benzodiazepines ${ }^{1,8}$, which exhibit reduced GABA-modulatory 
efficacy after $3 \alpha, 5 \alpha$-THP withdrawal as a result of upregulation of the GABA-receptor $\alpha 4$ subunit.

Sheryl S. Smith*, Qi Hua Gong*, Fu-Chun Hsu*, Ronald S. Markowitz ${ }^{\star}$, J. M. H. ffrench-Mullen $\dagger$, Xinshe $\mathrm{Li}^{*}$

*Department of Neurobiology and Anatomy, Allegheny University of the Health Sciences, EPPI, 3200 Henry Avenue, Philadelphia, Pennsylvania 19129, USA

$\dagger$ RIN Bioscience, Zeneca Pharmaceuticals, Wilmington, Delaware 19850-5437, USA

1. Smith, S. S. et al. Nature 392, 926-930 (1998)

2. Bjurulf, E., Selstam, G. \& Olofsson, J. I. J. Reprod. Fertil. 102, 139-145 (1994).

3. Uzunov, D. P., Cooper, T. B., Costa, E. \& Guidotti, A. Proc. Natl Acad. Sci. USA 93, 12599-12604 (1996).

4. Devaud, L. L., Purdy, R. H., Finn, D. A. \& Morrow, A. L. J. Pharmacol. Exp. Ther. 278, 510-517 (1996).

5. Devaud, L. L., Purdy, R. H. \& Morrow, A. L. Alcohol. Clin. Exp. Res. 19, 350-355 (1995).

6. Devaud, L. L., Fritschy, J. M., Sieghart, W. \& Morrow, A. L. J. Neurochem. 69, 126-130 (1997).

7. Costa, A. M., Spence, K. T., Smith, S. S. \& ffrench-Mullen, J. M. H. J. Neurophysiol. 74, 464-469 (1995).

8. Smith, S. S. et al. J. Neurosci. 18, 5275-5284 (1998).

9. Penning, T. M., Sharp, R. B. \& Krieger, N. R. J. Biol. Chem. 260, 15266-15272 (1985)

10. Bernardi, F. et al. Eur. J. Endocrinol. 138, 316-321 (1998).

\section{Young wallabies get a free ride}

Wallabies and kangaroos increase their speed of hopping with little increase in their use of metabolic energy, apparently by means of elastic energy savings in their hindlimb tendons ${ }^{1-3}$. Here we report that, because of this storage capacity, female tammar wallabies can carry large young in their pouch at very low cost, with no increase in metabolic energy expenditure.

In running mammals, including horses, dogs and rats, the addition of a load results in an increase in metabolic rate that is directly proportional to the load carried ${ }^{4}$. This extra power requirement is presumably linked to increases in mechanical work and to the development of greater force by the muscles. There are a few cases in which this pattern is not seen. Women of the Luo and Kikuyu tribes of Kenya can carry loads equivalent to $20 \%$ of their body weight with no measurable increase in metabolic rate, an offset that means they can carry loads of up to $70 \%$ of their body weight with less than the expected $70 \%$ increase in rate of oxygen consumption ${ }^{5,6}$. This efficiency is achieved with no increase in mechanical work, as the transfer between kinetic and potential energy components of walking is more complete ${ }^{7}$.

Unlike quadrupeds, in which faster speeds result in linear increases in the massspecific rate of oxygen consumption, wallabies and kangaroos can increase hopping speed without incurring further increases in metabolic $\operatorname{cost}^{1,2}$. This uncoupling of metabolic energy requirements and speed is believed to be achieved through the storage and recovery of elastic strain energy in the hindlimb tendons. Potential and kinetic energy lost on landing is stored and subsequently recovered from the tendons during each hop. In wallabies, elastic savings from the tendon represent a significant fraction of the measured metabolic power ${ }^{3,8}$.

In the tammar wallaby, Macropus eugenii, a mother weighing 4-5 kg carries its young in the pouch until its weight approaches 1 $\mathrm{kg}$. Four wallabies were trained to hop on a moving treadmill while carrying a load equivalent to $15 \%$ of their body weight. Because of the risk of losing young animals, the load consisted of lead shot placed in a deformable latex glove carried in the pouch. Metabolic rates were assessed from rates of oxygen consumption at two hopping speeds, 3.5 and $4.5 \mathrm{~m} \mathrm{~s}^{-1}$, in loaded and unloaded
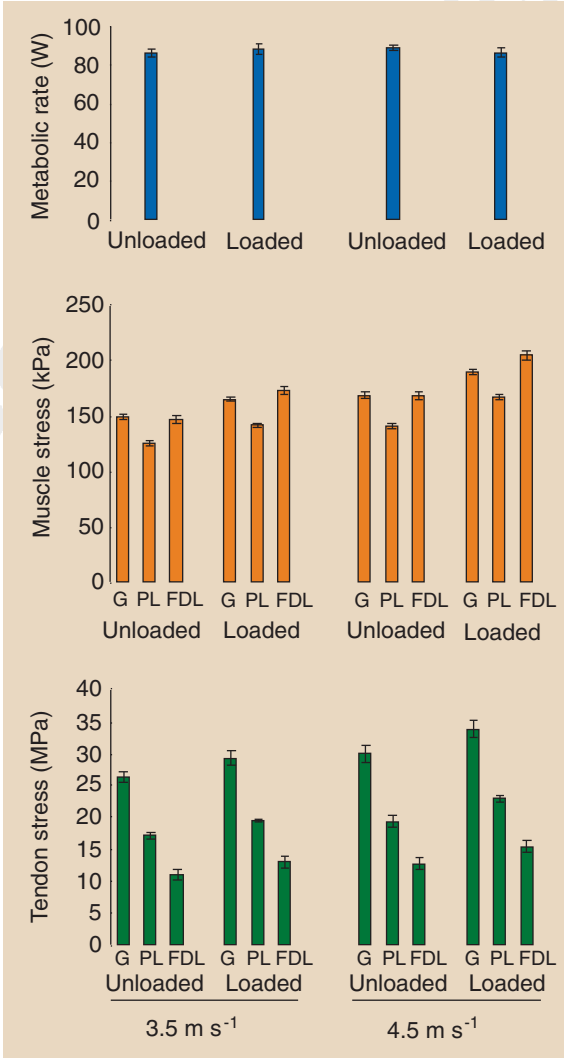

Figure 1 Metabolic rate and muscle and tendon stress in hopping wallabies. Top, metabolic rates, from oxygen consumption measured using a caloric equivalent whereby $1 \mathrm{ml} \mathrm{O}_{2}$ represents $20.1 \mathrm{~J}$, were measured using a flow-through transparent mask held in front of the hopping animal ${ }^{2}$. At the speeds selected, adding a load equivalent to $15 \%$ of body weight did not result in significant metabolic changes. Also shown are muscle (middle) and tendon (bottom) stresses, the force per unit crosssectional area ${ }^{3}$, in the gastrocnemius $(G)$, plantaris $(\mathrm{PL})$ and flexor digitorum longus ( $\mathrm{FDL}$ ) muscletendon units of four wallabies hopping on a moving treadmill. At both hopping speeds, the addition of the load resulted in significant increases in muscle and tendon stress in all three systems $(P<0.01)$. conditions $^{2}$. Direct force recordings were made from the three main ankle plantarflexor muscle-tendon systems by using stainless-steel buckle transducers ${ }^{3}$. The time the feet were on the ground and the hopping frequency were obtained indirectly from the recordings of force output.

Adding the load caused no significant change in metabolic rate during hopping, nor did it alter the stride frequency for a given speed, despite the increase in muscle and tendon stress. But adding the load did significantly increase the time for which the foot applied force to the ground during the stride. The duty factor (contact time/stride period) increased from 0.49 to 0.52 at both speeds. The percentage of metabolic power recovered by tendon strain energy increased from $7.2 \%$ to $9.2 \%$ with the addition of a $15 \%$ load at $3.5 \mathrm{~m} \mathrm{~s}^{-1}$; at $4.5 \mathrm{~m} \mathrm{~s}^{-1}$ this recovery rose from $9.3 \%$ to $12.9 \%$. This increase is less than the $15 \%$ increase in mass because the mechanical work saving is given as a percentage of metabolic energy cost. The relationship between these variables is not simple, particularly as duty factor changes. Wallabies carrying a load approximating the mass of a fully developed young have no increase in metabolic energy expenditure, and the increased muscle force required is offset by an increase in stored elastic strain energy. However, the time the foot was on the ground increased in the loaded condition. This might be expected to allow for the recruitment of slower muscle fibres, which could also help lower the energy cost ${ }^{9}$.

Many rodent species and some small birds have adopted a bipedal hopping gait, but kangaroos and wallabies are the only large vertebrates to do so. Given the apparent economies of this locomotory gait, and that the potential for tissue elasticity in muscle-tendon units is strongly mass dependent ${ }^{8}$, this is perhaps surprising. Our findings suggest that the evolution of a bipedal hopping gait has particular adaptive significance in animals that carry their young in a pouch until they reach a stage of advanced body weight. Kangaroos and wallabies may be the only group of animals in which carrying young is energetically 'free'.

R. V. Baudinette ${ }^{\star}$, A. A. Biewener $\dagger$

${ }^{\star}$ Department of Zoology,

University of Adelaide,

Adelaide, South Australia 5005, Australia

e-mail: rbaudinette@zoology.adelaide.edu.au

$\dagger$ Department of Organismal Biology and Anatomy, University of Chicago,

Chicago, Illinois 60637, USA

$\ddagger$ Present address: Concord Field Station,

MCZ Department of Organismic and Evolutionary Biology, Harvard University,

Bedford, Massachusetts 01730, USA

1. Dawson, T. J. \& Taylor, C. R. Nature 246, 313-314 (1973).

2. Baudinette, R. V .et al.. Am. J. Physiol. 262, 771-778 (1992).

3. Biewener, A. A. \& Baudinette, R. V. J. Exp. Biol. 198, 1829-1841 (1995). 